\title{
RE-CERTIFICATION OF HYDROXYVITAMIN D STANDARDS BY ISOTOPE PATTERN DECONVOLUTION
}

\author{
J. Pitarch-Motellón ${ }^{1}$, A.F. Roig-Navarro ${ }^{1}$, C. Le Goff ${ }^{2}$, E. Cavalier ${ }^{2}$ N. Fabregat- \\ Cabello ${ }^{2^{*}}$. \\ ${ }^{1}$ Research Institute for Pesticides and Water, Universitat Jaume I, Castelló, Spain
}

${ }^{2}$ Department of Clinical Chemistry, University of Liège, CHU Sart-Tilman, Liège, Belgium

*Author Present address

Neus Fabregat-Cabello, Hematology Research group, Health Research Institute Hospital La $\mathrm{Fe}$ (IIS La Fe), Valencia, Spain

${ }^{*}$ Corresponding author:

Neus Fabregat-Cabello, Ph.D., Hematology Research group, Health Research Institute Hospital La Fe (IIS La Fe), Valencia, Spain, Phone: +34 9612 46692,Fax: +34 961246620 , E-mail: N.Fabregat@outlook.es

\section{Keywords}

LC-MS/MS; isotope dilution mass spectrometry; vitamin D; certification; isotope pattern deconvolution; quality control 


\section{Abstract}

Background: Vitamin D testing in analytical clinical laboratories has been experiencing a rapid increase of demand over the last years, as it plays a key role in several disorders. Due to the narrow ranges of medical significance regarding its concentration levels in human serum, accurate and precise determinations of vitamin D metabolites are required

Methods: We present an isotope dilution mass spectrometry quantification method for the re-certification of routine commercial standards used in method validation steps, isotope pattern deconvolution (IPD) based on LC-MS/MS.

Results:.IPD allowed to compensate for the observed biases of $+4.7 \%$ for $25(\mathrm{OH}) \mathrm{D} 3$, $-29 \%$ for $25(\mathrm{OH}) \mathrm{D} 2$ and $-30 \%$ for $24,25(\mathrm{OH})_{2} \mathrm{D}_{3}$ standard concentrations, respectively in an easy, cheap and straightforward way.

Conclusions: Is has been observed that, in some cases, discrepancies may exist between stated purity or amount of routinely used commercial standards and actual values, which would lead to unwanted bias in the developed methodologies. The present correction has helped meeting the regulations established by international standardization programs, including Vitamin D Standardization Program (VDSP). 


\section{Introduction}

Vitamin D (VTD) is an important prohormone widely known since its deficiency is directly related to development of rickets in children and osteoporosis in adults. Furthermore, recent studies have demonstrated that vitamin $D$ has also an important role in non-skeletal conditions such as autoimmune diseases, cardiovascular diseases and cancer, among others[1]. This vitamin can be found in two main forms: vitamin D2 and vitamin D3. The most common form is vitamin D3 (or cholecalciferol) and can be obtained either from the diet (mostly fatty fish) or endogenous biosynthesis under the effect of UV rays of sunlight. The other form, vitamin D2 (ergocalciferol or calciferol) is derived from ergosterol, a yeast sterol found in mushrooms. The metabolism of both forms of vitamin $D$ are subjected to a first hydroxylation in the liver to form 25-hydroxyvitamin $D(25(\mathrm{OH}) \mathrm{D})$ and then to a second one in the kidney to form 1,25-dihydroxyvitamin $D\left(1,25(\mathrm{OH})_{2} \mathrm{D}\right)$, the active form of vitamin D. Nevertheless, the measurement of $25(\mathrm{OH}) \mathrm{D}$ in serum samples is preferred test for the assessment of vitamin D status over the $1,25(\mathrm{OH})_{2} \mathrm{D}$. There are two main reasons for this choice: the longer lifetime ( 3 weeks versus 4 h) and its higher concentration levels $(\mathrm{ng} / \mathrm{mL}$ versus $\mathrm{pg} / \mathrm{mL})[2]$. Besides, some other VTD metabolites have gained more attention in the last years. Among them, $24,25(\mathrm{OH})_{2} \mathrm{D}_{3}$, should be mentioned due to its correlation with $25(\mathrm{OH}) \mathrm{D}_{3}$ which can help to assess VTD status (3).

Over the last years, a dramatic rise in vitamin $D$ testing (as $25(\mathrm{OH}) \mathrm{D})$ has been observed due to different reasons [3-5]. The assays currently available for this determination can be classified into binding (mostly automatic immunoassays) and chemical assays (HPLC and LC-MS/MS) [6,7]. As a consequence, and due to the poor agreement observed among all those different assays an urgent need of 
standardization across the different methods is needed in order to assure a correct diagnostic and treatment of the patients. Furthermore, the traditional 25(OH)D cutoffs in use for vitamin $D$ deficiency are either 20 or $30 \mathrm{ng} / \mathrm{mL}$, and have been established using Radiolmmuno Assays (RIA). In order to deal with the problem of lack of agreement between assays, different entities with international reputation have created programs to establish world-wide standardization, including the VDSP (Vitamin D Standardization Program) from the United States and the DEQAS (Vitamin D External Quality Assessment Scheme) from the United Kingdom. According to the VDSP recommendations, a total $\mathrm{CV} \leq 10 \%$ and a maximum mean bias against a reference method of $\leq 5 \%$ is accepted for the method under standardization [8].

There are several factors that can result in inadequate values for routine precision and bias. Among them, those related to method unspecificity, instrumental biased measurements, and calibration solution issues [9-12]. The last one, working with reliable standard solutions, is recognised as a key problem regardless of the analytical methodology used. Usual problems with commercial calibration solution or standards are stability and concentration not accurately certified $[9,10,13,14]$. In this way, it is recommended to periodically check the concentration of vitamin D isomers. A straightforward way to deal with this problem is to buy, if available, certified calibrators from prestigious entities, e.g. the National Institute of Standards and Technology (NIST). However, routine use of high quality standards is not always the best option due to their usually unattainable price. Thus, recertifying calibrator solutions can be a good choice.

Mass spectrometry is currently the method of choice for the determination of thermolabile or non-volatile compounds. Despite its known advantages, sample 
measurements are not free of fluctuations, either by instrument variation (injection volume, detector fluctuations, etc.) or by the composition of samples (mostly matrix effects able to modify of the ionization in the electrospray). All these problems can be compensated by the addition of an Isotopically-Labelled Internal Standard (ILIS). This method is called isotope dilution mass spectrometry (IDMS) and is known to yield the most accurate, precise and reliable results [15]. The most common approach is to create a calibration curve by plotting the ratio of the analyte signal to the internal standard signal as a function of the analyte concentration standards, when a constant amount of ILIS is added to both samples and calibrators. A second approach is the quantification based on Isotope Pattern Deconvolution, which makes use of the artificial alteration of the natural isotopomer abundances when a known amount of a labelled analogue is added to a sample. Calculations are based on multiple linear regression without the use of methodological calibration, thus reducing the total analysis time. Being an IDMS based method, IPD results are traceable to the International System of units $(\mathrm{SI})$ [16-18]. This reliable approach has been satisfactorily tested for rapid quantifications in different complex matrices, such as urine, food and environmental samples [19-22].

In this work we show a re-certification approach for $25(\mathrm{OH}) \mathrm{D}_{2} / \mathrm{D}_{3}$ and $24,25(\mathrm{OH})_{2} \mathrm{D}_{3}$ solvent standards based on Isotope Pattern Deconvolution using NIST SRM 2971 and 2972 as reference materials. With this approach, that faces the core problem of poor certification of some standards, the exact concentration of commercial standards are accurately known. Thus, the analytical method meets the requirements for external standardization criteria using in-house calibration curves. 


\section{Materials and Methods}

\section{$\underline{\text { Materials }}$}

Standards of 25-hydroxyvitamin $\mathrm{D}_{2}\left(25(\mathrm{OH}) \mathrm{D}_{2}\right),{ }^{2} \mathrm{H}_{3}-25$-hydroxyvitamin $\mathrm{D}_{2}\left({ }^{2} \mathrm{H}_{3}-\right.$ $\left.25(\mathrm{OH}) \mathrm{D}_{2}\right)$ and 24,25-dihydroxyvitamin $\mathrm{D}_{3}\left(24,25(\mathrm{OH})_{2} \mathrm{D}_{3}\right)$ were purchased from Isosciences (K. of Prussia, PA, USA). Standards of 25-hydroxyvitamin $D_{3}$ (25(OH)D $\left.\mathrm{D}_{3}\right),{ }^{2} \mathrm{H}_{6}-25$-hydroxyvitamin $\mathrm{D}_{3}\left({ }^{2} \mathrm{H}_{6}-25(\mathrm{OH}) \mathrm{D}_{3}\right)$ and ${ }^{2} \mathrm{H}_{6}-24,25$-hydroxyvitamin $\mathrm{D}_{3}\left({ }^{2} \mathrm{H}_{6}-24,25(\mathrm{OH})_{2} \mathrm{D}_{3}\right)$ were from Medical Isotopes (Pelham, NH, USA). SRM2972 solvent-based materials for $25(\mathrm{OH}) \mathrm{D}_{2}, 25(\mathrm{OH}) \mathrm{D}_{3}(1$ level) and SRM2971 solventbased materials for $24,25(\mathrm{OH})_{2} \mathrm{D}_{3}$ were purchased from the National Institute for Standards and Technology (NIST, Gaithersburg, MD, USA). All stock solutions were stored at $-20^{\circ} \mathrm{C}$ and employed to prepare daily gravimetrically diluted working standard solutions in methanol. MSG2000 Vitamin D-free human serum was purchased from Golden West Biological (Temecula, CA, USA). Structures of each vitamin $\mathrm{D}$ forms can be consulted in Table 1.

Individual stock solutions of $25(\mathrm{OH}) \mathrm{D}_{2},{ }^{2} \mathrm{H}_{3}-25(\mathrm{OH}) \mathrm{D}_{2}, 25(\mathrm{OH}) \mathrm{D}_{3},{ }^{2} \mathrm{H}_{6}-25(\mathrm{OH}) \mathrm{D}_{3}$, $24,25(\mathrm{OH})_{2} \mathrm{D}_{3}$ and ${ }^{2} \mathrm{H}_{6}-24,25(\mathrm{OH})_{2} \mathrm{D}_{3}$ were prepared gravimetrically by weighting the volume of methanol added. In all cases the certified amount of analyte was $1 \mathrm{mg}$, giving approximate concentrations of $1 \mathrm{mg} / \mathrm{mL}$. Theoretical exact concentrations were obtained taking into account the purity percentage as indicated in the Certificate of Analysis (CoA). Sequential dilutions were also prepared by weight in methanol at a concentration level of 50,10 and $0.5 \mu \mathrm{g} / \mathrm{mL}$. Exact values are finally recertified using IPD calculations and SRM 2971 and 2972 as reference standards (see results section). 
Methanol, acetonitrile and water were all LC-MS grade from Biosolve BV (Valkenswaard, Netherlands). Formic acid additive for LC-MS was purchased from Fisher Chemicals (Loughborough, UK).

\section{$\underline{\text { LC-MS/MS Conditions }}$}

Identification and quantification of the selected compounds was carried out with an UHPLC system using a Nexera X2 UPLC (Shimadzu, Kyoto, Japan) interfaced to a quadrupole-linear ion trap QTRAP 6500 system from AB Sciex (Foster City, CA, USA) using an lonDrive ${ }^{\mathrm{TM}}$ Turbo $\mathrm{V}$ ion source. Chromatographic separation was performed on a pentafluorophenyl Kinetex PFP $100 \AA$ column $(100 \times 2.1 \mathrm{~mm}, 2.6 \mu \mathrm{m})$ fitted with a UPLC SecurityGuard ULTRA cartridge from Phenomenex (Torrance, CA, USA). The mobile phase was a time programmed gradient using $A$ (water) and $B$ (methanol), both modified with $0.1 \%$ formic acid. Starting from $40 \%$ methanol a final $95 \%$ was reached. Analytes were ionized by atmospheric pressure chemical ionization in positive mode (APCI+) and detected by MS/MS in Selected Reaction Monitoring (SRM). Further information can be consulted elsewhere [23].

Analyst v1.6.2 (Sciex) software was used for data acquisition. Data treatment for quantitation was performed on MultiQuant v3.0.2 (Sciex).

\section{Isotope Pattern Deconvolution}

IPD is an alternative to classical Isotope Dilution Mass Spectrometry (IDMS) calibration procedures. that avoids the preparation of a calibration curve. It is an isotope dilution mass spectrometry quantification method which is based on the 
multiple linear regression calculation, after the addition of isotopically enriched analogues of the analytes under study, producing an alteration of the natural distribution of isotopic abundances. Briefly, the resulting isotopic composition $\left(A_{m i x}^{S R M_{i}}\right)$, (Figure 1C) measured after mixing the natural occurring analytes in the samples and the spiked labelled analogues, is a linear combination of the natural $\left(A_{n a t}^{S R M_{i}}\right)$ (Figure $1 \mathrm{~A})$ and enriched $\left(A_{\text {lab }}^{S R M_{i}}\right)$ (Figure 1B) compound's isotopic distribution of abundances. The generic isotopic distribution also shows possible impurities in the labelled compound due to improper or incomplete labelling of the structure (Figure 1B). Using tandem MS in the SRM mode isotopic abundances can be experimentally measured from the transitons generated by the parent ion. Thus, for $n$ measured transitions in the MS/MS system, this can be expressed as matrix notation as follows:

$$
\left[\begin{array}{c}
A_{\text {mix }}^{S R M_{1}} \\
A_{\text {mix }}^{S R M_{2}} \\
\vdots \\
A_{\text {mix }}^{S R M_{n}}
\end{array}\right]=\left[\begin{array}{cc}
A_{\text {nat }}^{S R M_{1}} & A_{\text {lab }}^{S R M_{1}} \\
A_{\text {nat }}^{S R M_{2}} & A_{\text {lab }}^{S R M_{2}} \\
\vdots & \vdots \\
A_{\text {nat }}^{S R M_{n}} & A_{\text {lab }}^{S R M_{n}}
\end{array}\right]\left[\begin{array}{c}
X_{\text {nat }} \\
X_{\text {lab }}
\end{array}\right]+\left[\begin{array}{c}
e^{S R M_{1}} \\
e^{S R M_{2}} \\
\vdots \\
e^{S R M_{n}}
\end{array}\right] \text { (Eq. 1) }
$$

Individual abundances for natural $\left(A_{\text {nat }}^{S R M_{i}}\right)$ and labeled $\left(A_{\text {lab }}^{S R M_{i}}\right)$ analogues must be obtained measuring the whole cluster transtions. Final calculations only need a minimum of three measured transitions in the spiked sample $\left(A_{\text {mix }}^{S R M_{i}}\right)$ [16]. In order to solve the equation system, an error vector is included $\left(e^{S R M_{i}}\right)$. Minimization of this parameter by multiple linear regression gives the values of molar fractions $\left(X_{\text {nat }}\right.$ and $X_{\text {lab }}$ respectively) that best fit the experimental data of the mix. These calculations can be easily performed with the Excel Linest function, which uses the least squares method to calculate the line of best fit through a supplied set of $y$ (in this cases, $\left.A_{\text {mix }}^{S R M_{i}}\right)$ and $\mathrm{x}\left(\left(A_{\text {nat }}^{S R M_{i}}\right)\right.$ and $\left.\left(A_{\text {lab }}^{S R M_{i}}\right)\right)$ values. Finally, since the amount of labelled 
compound $\left(N_{\text {lab }}\right)$ spiked into the sample is known, the amount of natural compound $\left(N_{\text {nat }}\right)$ is readily obtained:

$N_{\text {nat }}=N_{\text {lab }} \frac{X_{\text {nat }}}{X_{\text {lab }}}($ Eq. 2)

As said before, no methodological calibration is needed, and quantification is directly conducted in the sample after one injection. This methodology requires the full characterization of both compounds (natural and labelled) in terms of their isotopomer distribution of abundances. In the present work isotopomer abundances have been experimentally measured. In addition, the exact concentration of the labelled standard solutions should be obtained by reverse isotope dilution (RID), using the labelled standard, as a sample, and the certified SRM standards. (See Supplemental Data, IPD_detailed section, for a more detailed explanation and an example of calculation)

\section{Results}

As a first step, in the framework a VTD study, solvent based reference materials were analysed, using external calibration, with the commercial standards available in the laboratory. Accuracy of results was unacceptable. Bias was near $30 \%$ for some of the metabolites determined. Thus, commercial standards were recertified using IDMS and IPD calculations, a tested fast, reliable and traceable methodology. The whole procedure includes the exact determination of the concentration of the labelled compounds by Reverse Isotope Dilution (RID) using the NIST SRM 2972 and SRM 2971 as reference materials and the subsequent calculation of the exact concentration of the commercial natural standards by IPD. 
As said above, IPD provides a fast and reliable way to quantify analyte concentrations without the need of performing methodological calibrations while it is directly traceable to the International System of Units. Nevertheless, it is required a careful and extensive characterization of natural and labelled compounds. That is, isotopic distribution of abundances needs to be accurately known as well as the concentration of labelled solutions used for spiking samples, which is obtained by the aforementioned RID methodology.

Detailed and extended information of IPD quantification methodology can be found in the bibliography [16-18].

\section{Characterization of abundances}

Once the suitable transitions were selected, average experimental abundances were obtained by injection of $0.5 \mu \mathrm{g} / \mathrm{mL}$ individual standards for each natural and labelled compounds $(n=5)$. Enhanced Product lon (EPI) acquisition mode was used in order to get the necessary mass resolution to avoid peak overlaping. An example of spectrum acquisition can be observed in Figure 2. Results are shown on Supplemental Data Tables 1, 2 and 3.

\section{$\underline{\text { Reverse Isotope Dilution (RID) of labelled compounds }}$}

As exposed before, the proposed approach of IPD requires the determination of the exact concentration of the labelled compound. For this purpose, $0.04 \mathrm{~g}$ of each isotopically labelled compound solution was individually mixed with also $0.04 \mathrm{~g}$ of certified solution of its natural analogue, either SRM2972 $\left(25(\mathrm{OH}) \mathrm{D}_{3}\right.$ and $\left.25(\mathrm{OH}) \mathrm{D}_{2}\right)$ 
or SRM2971 $\left(24,25(\mathrm{OH})_{2} \mathrm{D}_{3}\right)$ solvent-based material and diluted with $0.1 \mathrm{~g}$ of water. Final solutions of approximately $500 \mathrm{ng} / \mathrm{g}$ of both compounds were gravimetrically prepared in triplicate, and injected in the LC-MS/MS system also in triplicate and finally determined by RID mass spectrometry. Table 2 summarizes the results obtained for the labelled compound solutions of $10 \mu \mathrm{g} / \mathrm{mL}$ of nominal concentration for $25(\mathrm{OH}) \mathrm{D}$ and $1 \mu \mathrm{g} / \mathrm{mL}$ for $24,25(\mathrm{OH})_{2} \mathrm{D}_{3}$, prepared by gravimetric dilutions of the stock solutions. Exact concentrations show high precision with $\mathrm{CV}<2.5 \%$.

\section{Re-certification of the natural standards by IPD}

A similar approach to that used in RID was employed for IPD calculations. In this case, the real concentrations of the natural analogues were calculated with the exact concentration obtained for the labelled counterparts. Individual IPD solutions were prepared gravimetrically mixing $0.04 \mathrm{~g}$ of RID certified labelled solution with $0.04 \mathrm{~g}$ of natural solutions. Solutions were prepared in triplicate, and injected in the LC-MS/MS system also in triplicate and finally determined by IPD mass spectrometry.

For $25(\mathrm{OH}) \mathrm{D}_{3}$, the concentration obtained by IPD, $732 \mu \mathrm{g} / \mathrm{mL}$, was in line with the theoretical values of $765 \mu \mathrm{g} / \mathrm{mL}$. In the case of $25(\mathrm{OH}) \mathrm{D}_{2}$, the concentration obtained by IPD was $1258 \mu \mathrm{g} / \mathrm{mL}$ while the theoretical concentration, according to the commercial certificate, was $976 \mu \mathrm{g} / \mathrm{mL}$. Similar results were obtained for $24,25(\mathrm{OH})_{2} \mathrm{D}_{3}($ Table 3$)$. These results mean a bias of $-4.7 \%$ for the calibrator of $25(\mathrm{OH}) \mathrm{D}_{3}$, a bias of $+29 \%$ in the case of $25(\mathrm{OH}) \mathrm{D}_{2}$ and a bias of $+30 \%$ for $24,25(\mathrm{OH})_{2} \mathrm{D}_{3}$. As a consequence, the use of solutions prepared without any previous re-certification would imply a systematic bias of $+4.7 \%,-29 \%$ and $-30 \%$ in the determination of real samples. 
Results obtained for commercial (not CRMs) standards, natural and labelled, point out the need to recertify any stock standard solutions (against a suitable CRM), thus, avoiding problems of inaccuracy of commercial certifications or degradation processes, and unreliable final results.

\section{Comparison with reference samples from the VDSP program}

For comparison and validation purposes, we tested 80 reference samples provided by the VDSP program and compared them to the real total vitamin D value using the reference method [23]. Quantification of samples was performed with the corrected values obtained for the standards by IPD. An average bias of $3 \%$ was obtained which is in accordance with the VDSP recommendations that accept a $\pm 5 \%$ bias.

\section{Discussion}

The results of the current study show the importance of recertify the concentration of critical standards. In addition, the suitability of Isotope Pattern Deconvolution, a tested fast and reliable method, to provide accurate diagnostics is demonstrated. The present study demonstrates the high impact that may result from the lack of knowledge of the exact values, where a simple erroneous concentration can originate high loses in the clinical laboratory. Besides, these biases are usually attributed to sample preparation instead of standard concentration.

As stated in the introduction, Isotope Pattern Deconvolution has been applied for the accurate quantification of a great variety of samples, including environmental, food and human samples. Besides some clinical applications have also been developed $[19,24]$ but not a single re-certification strategy has been described before. 
The present approach permits also buying cheaper standards with either unknown concentrations or low purity and recertify their concentration against international recognised standards, directly traceable to the international system of units. Another advantage of the recertification is that it avoids the repetition of sample analyses when the systematic bias has been detected and studied. Finally, it permits to retest periodically the laboratory standards with high-level standards.

When very strict quality controls are required the concentration provided by these standards may be unacceptable. Positive bias in the concentration can be attributed to an error in the weighting by the seller or evaporation of the solvent used for the solution, while negative bias could be explained by a loss of the solid standard, including degradation.

Another possibility is the fact that some vendors include on the vial label " $1 \mathrm{mg}$ ", being this value in some cases an approximate weighting. As a consequence, it is absolutely necessary to verify with the vendor before the shipping of the material if this value corresponds to an exact weighting. Usually, an accurate weight will increase the standard price around a $5-10 \%$ of the total price.

In conclusion, in this study we have demonstrated the power of Isotope Pattern Deconvolution for the easy and accurate re-certification of vitamin D standards. In addition, we have shown that the preparation of solvent standards from vials containing, in theory, $1 \mathrm{mg}$ of solid can be a simple yet an important source of error. Re-certification by IPD using a reference standard in solvent is able to correct these problems in a fast, reliable and cost-saving way with the highest confidence. 


\section{Conflicts of interest}

There are no conflicts to declare.

\section{Acknowledgements}

This research did not receive any specific grant from funding agencies in the public, commercial, or not-for-profit sectors.

\section{References}

[1] R.M. Gathungu, C.C. Flarakos, G.S. Reddy, P. Vouros, THE ROLE OF MASS SPECTROMETRY IN THE ANALYSIS OF VITAMIN D COMPOUNDS, MasS Spectrom. Rev. 32 (2013) 72-86. doi:10.1002/mas.

[2] M.L. Musteata, F.M. Musteata, Overview of extraction methods for analysis of vitamin D and its metabolites in biological samples, Bioanalysis. 3 (2011) 1987-2002. doi:10.4155/bio.11.195.

[3] J.M.W. van den Ouweland, Analysis of vitamin D metabolites by liquid chromatography-tandem mass spectrometry, TrAC Trends Anal. Chem. 84 (2016) 117-130. doi:10.1016/j.trac.2016.02.005.

[4] S.H. Atef, Vitamin D assays in clinical laboratory: Past, present and future challenges, J. Steroid Biochem. Mol. Biol. 175 (2018) 136-137. doi:10.1016/j.jsbmb.2017.02.011.

[5] N. Sattar, Biomarkers for diabetes prediction, pathogenesis or pharmacotherapy guidance? Past, present and future possibilities, Diabet. Med. 29 (2012) 5-13. doi:10.1111/j.1464-5491.2011.03480.x.

[6] J.M.W. van den Ouweland, Analysis of vitamin D metabolites by liquid 
chromatography-tandem mass spectrometry, TrAC Trends Anal. Chem. 84 (2016) 117-130. doi:10.1016/j.trac.2016.02.005.

[7] S.H. Atef, Vitamin D assays in clinical laboratory: Past, present and future challenges, J. Steroid Biochem. Mol. Biol. 175 (2018) 136-137. doi:10.1016/j.jsbmb.2017.02.011.

[8] C.T. Sempos, J.M. Betz, J.E. Camara, G.D. Carter, E. Cavalier, M.W. Clarke, K.G. Dowling, R.A. Durazo-Arvizu, A.N. Hoofnagle, A. Liu, K.W. Phinney, K. Sarafin, S.A. Wise, P.M. Coates, General steps to standardize the laboratory measurement of serum total 25-hydroxyvitamin D, J. AOAC Int. 100 (2017) 1230-1233. doi:10.5740/jaoacint.17-0259.

[9] M. Bedner, K.A. Lippa, 25-Hydroxyvitamin D isomerizes to pre-25hydroxyvitamin D in solution: considerations for calibration in clinical measurements, Anal. Bioanal. Chem. 25 (2015) 8079-8086. doi:10.1007/s00216-015-8980-z.

[10] G.D. Carter, 25-Hydroxyvitamin D assays: The quest for accuracy, Clin. Chem. 55 (2009) 1300-1302. doi:10.1373/clinchem.2009.125906.

[11] E.A. Yetley, C.M. Pfeiffer, R.L. Schleicher, K.W. Phinney, D.A. Lacher, S. Christakos, J.H. Eckfeldt, J.C. Fleet, G. Howard, A.N. Hoofnagle, S.L. Hui, G.L. Lensmeyer, J. Massaro, M. Peacock, B. Rosner, D. Wiebe, R.L. Bailey, P.M. Coates, A.C. Looker, C. Sempos, C.L. Johnson, M.F. Picciano, NHANES monitoring of serum 25-hydroxyvitamin D: a roundtable summary, J. Nutr. 140 (2010) 2030S-2045S. doi:10.3945/jn.110.121483.

[12] D.A. Volmer, L.R.B.C. Mendes, C.S. Stokes, Analysis of vitamin D metabolic markers by mass spectrometry: current techniques, limitations of the "Gold standard" method, and anticipated future directions, Mass Spectrom. Rev. 34 
(2015) 2-23. doi:10.1002/mas.

[13] G.D. Carter, J.C. Jones, Use of a common standard improves the performance of liquid chromatography-tandem mass spectrometry methods for serum 25hydroxyvitamin-D, Ann. Clin. Biochem. 46 (2009) 79-81. doi:10.1258/acb.2008.008135.

[14] G. D. Carter, Accuracy of 25-Hydroxyvitamin D Assays: Confronting the Issues, Curr. Drug Targets. 12 (2011) 19-28. doi:10.2174/138945011793591608.

[15] A. Furey, M. Moriarty, V. Bane, B. Kinsella, M. Lehane, lon suppression; A critical review on causes, evaluation, prevention and applications, Talanta. 115 (2013) 104-122. doi:10.1016/j.talanta.2013.03.048.

[16] J.I. García Alonso, P. Rodríguez-González, Isotope Dilution Mass Spectrometry, Royal Society of Chemistry, 2013.

[17] A. González-Antuña, P. Rodríguez-González, G. Centineo, J.I. García Alonso, Evaluation of minimal 13C-labelling for stable isotope dilution in organic analysis, Analyst. 135 (2010) 953. doi:10.1039/b924432h.

[18] Á. Castillo, E. Gracia-Lor, A.F. Roig-Navarro, J. Vicente Sancho, P. RodríguezGonzález, J.I. García Alonso, Isotope pattern deconvolution-tandem mass spectrometry for the determination and confirmation of diclofenac in wastewaters., Anal. Chim. Acta. 765 (2013) 77-85.

[19] A. González-Antuña, P. Rodríguez-González, G. Centineo, J.I. García Alonso, Simultaneous determination of seven $\beta 2$-agonists in human and bovine urine by isotope dilution liquid chromatography-tandem mass spectrometry using compound-specific minimally 13C-labelled analogues, J. Chromatogr. A. 1372 (2014) 63-71. doi:10.1016/j.chroma.2014.10.065.

[20] J. Pitarch-Motellón, J. V. Sancho, M. Ibáñez, O. Pozo, A.F. Roig-Navarro, 
Determination of selected endogenous anabolic androgenic steroids and ratios in urine by ultra high performance liquid chromatography tandem mass spectrometry and isotope pattern deconvolution, J. Chromatogr. A. 1515 (2017) 172-178. doi:10.1016/j.chroma.2017.08.006.

[21] N. Fabregat-Cabello, P. Zomer, J.V. Sancho, A.F. Roig-Navarro, H.G.J. Mol, Comparison of approaches to deal with matrix effects in LC-MS/MS based determinations of mycotoxins in food and feed, World Mycotoxin J. 9 (2016) 149-161. doi:10.3920/WMJ2014.1872.

[22] N. Fabregat-Cabello, J. Pitarch-Motellón, J.V. Sancho, M. Ibáñez, A.F. RoigNavarro, Method development and validation for the determination of selected endocrine disrupting compounds by liquid chromatography mass spectrometry and isotope pattern deconvolution in water samples. Comparison of two extraction techniques, Anal. Methods. 8 (2016) 2895-2903. doi:10.1039/C6AY00221H.

[23] N. Fabregat-Cabello, J. Farre-Segura, L. Huyghebaert, S. Peeters, C. Le Goff, J.-C.C. Souberbielle, E. Cavalier, A fast and simple method for simultaneous measurements of $25(\mathrm{OH}) \mathrm{D}, 24,25(\mathrm{OH}) 2 \mathrm{D}$ and the Vitamin D Metabolite Ratio (VMR) in serum samples by LC-MS/MS, Clin. Chim. Acta. 473 (2017) 116-123. doi:10.1016/j.cca.2017.08.024.

[24] M. Fernández-Fernández, P. Rodríguez-González, M.E. Añón Álvarez, F. Rodríguez, F.V.Á. Menéndez, J.I.G. Alonso, Simultaneous Determination of Creatinine and Creatine in Human Serum by Double-Spike Isotope Dilution Liquid Chromatography-Tandem Mass Spectrometry (LC-MS/MS) and Gas Chromatography-Mass Spectrometry (GC-MS), Anal. Chem. 87 (2015) 37553763. doi:10.1021/acs.analchem.5b00769. 


\section{Tables}

Table 1. Structures of natural and labelled 25-hydroxyvitamin D2 and D3 and 24,25dihydroxyvitamin D3.

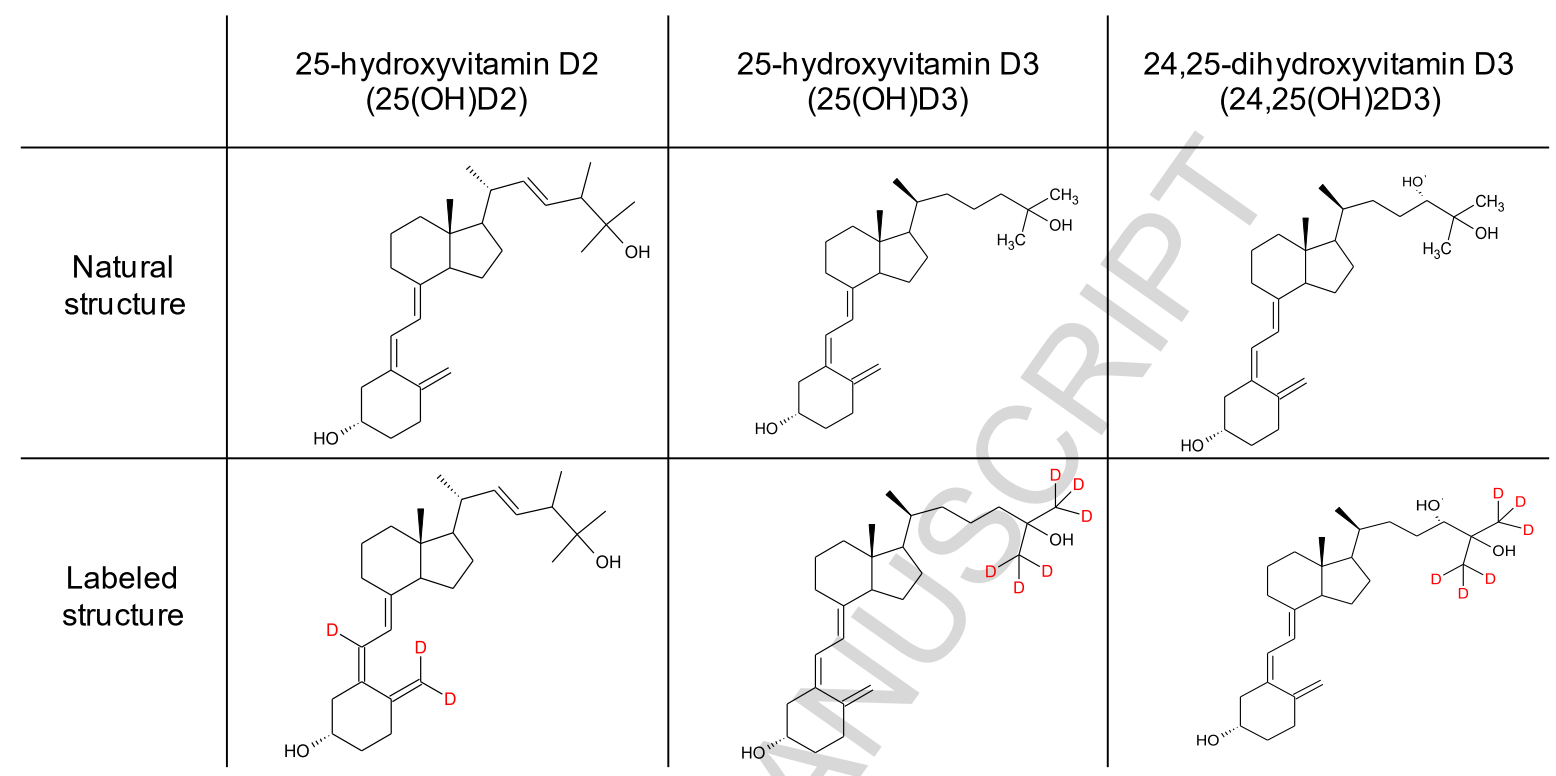


Table 2. Experimentally calculated concentration of labelled standards used for spiking, determined by Reverse Isotope Dilution (RID).

\begin{tabular}{cccc}
\hline Compound & Mean $^{1}(\mu \mathrm{g} / \mathrm{mL})$ & $\mathrm{SD}^{2}$ & RSD \\
\hline${ }^{2} \mathrm{H}_{3}-25(\mathrm{OH}) \mathrm{D} 2$ & 7.17 & 0.07 & $1.0 \%$ \\
${ }^{2} \mathrm{H}_{6}-25(\mathrm{OH}) \mathrm{D} 3$ & 5.12 & 0.13 & $2.5 \%$ \\
${ }^{2} \mathrm{H}_{6}-24,25(\mathrm{OH}) 2 \mathrm{D} 3$ & 1.340 & 0.012 & $0.9 \%$ \\
\hline
\end{tabular}

${ }^{1}$ Theoretical nominal concentration should be $10 \mu \mathrm{g} / \mathrm{mL}$ for ${ }^{2} \mathrm{H}_{3}-25(\mathrm{OH}) \mathrm{D} 2$ and ${ }^{2} \mathrm{H}_{6^{-}}$ 25(OH)D3 and $1 \mu \mathrm{g} / \mathrm{mL}^{2} \mathrm{H}_{6}-24,25(\mathrm{OH}) 2 \mathrm{D} 3$.

${ }^{2} \mathrm{SD}=$ Standard Deviation, RSD=Relative Standard Deviation. $\mathrm{n}=3$, injected three times each 
Table 3. Re-certification of natural standards by IPD using their labelled analogues.

\begin{tabular}{|c|c|c|c|c|}
\hline Compound & $\begin{array}{c}\text { Theoretical } \\
\text { concentration }(\mu \mathrm{g} / \mathrm{mL})\end{array}$ & Mean $(\mu \mathrm{g} / \mathrm{mL})$ & $\mathrm{SD}^{1}$ & RSD \\
\hline $25(\mathrm{OH}) \mathrm{D} 2$ & 976 & 1258 & 8 & $0.7 \%$ \\
\hline 25(OH)D3 & 765 & 732 & 9 & $1.2 \%$ \\
\hline $24,25(\mathrm{OH}) 2 \mathrm{D} 3$ & 933 & 1210 & 13 & $1.1 \%$ \\
\hline
\end{tabular}




\section{Figure captions}

Figure 1 Generic illustration of the IPD process including the isotopic distribution of natural $(A)$ and labelled compounds (B) and the corresponding combined distribution assuming a 1:1 mixture of both analogues (C) for five transitions in Selected Reaction Monitoring (SRM).

Figure 2. Enhanced Product lon acquisition for 24,25(OH)2D3. 


\section{Highlights}

- Accurate and precise determinations of vitamin D metabolites are required.

- Current commercially available standards (not CRMs) are not always properly characterized.

- Re-certification of those routinely used standards was performed by isotope pattern deconvolution. 


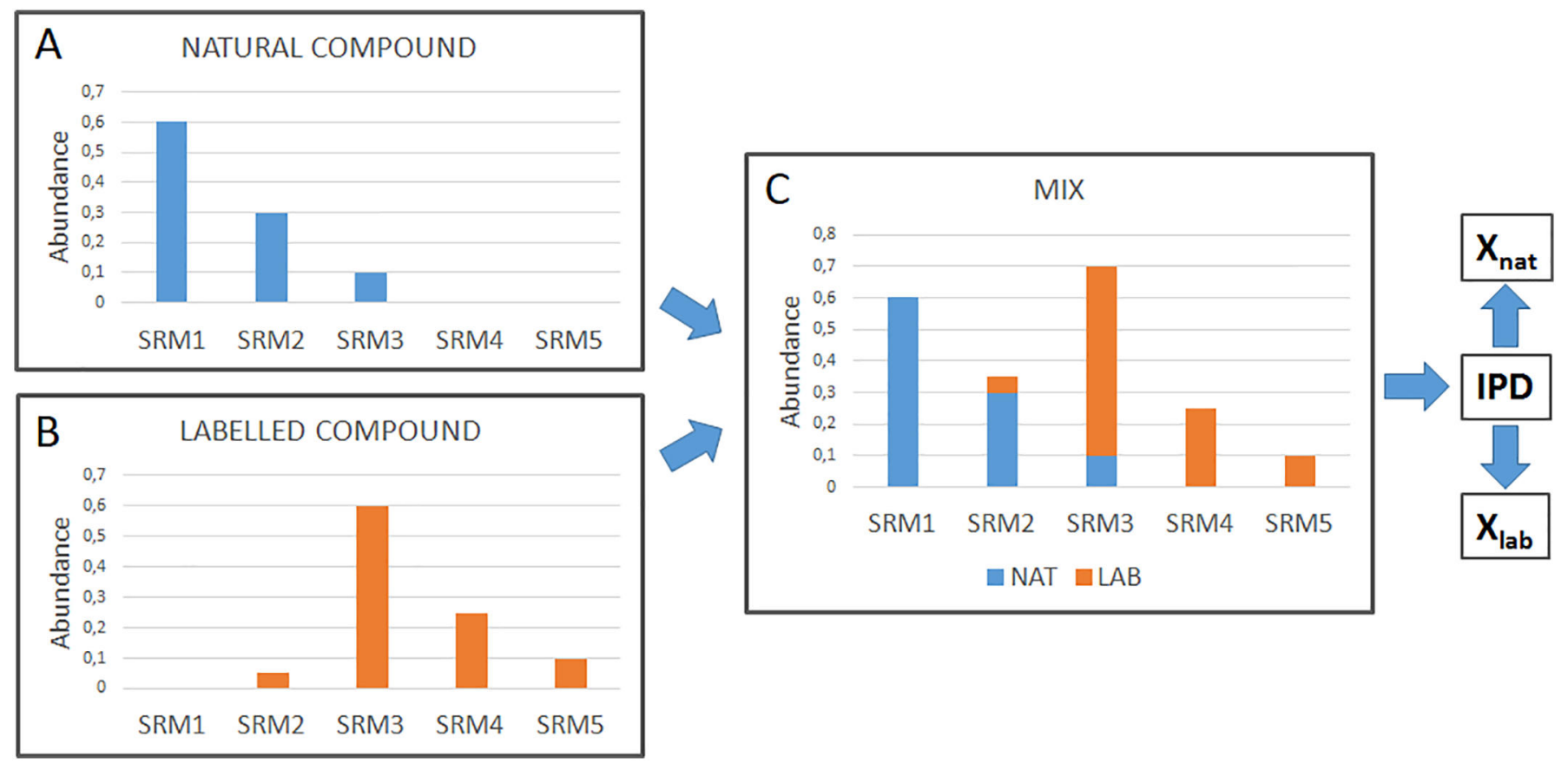

Figure 1 
- +ER: 2.387 to $2.576 \mathrm{~min}$ from Sample 7 (ER_24,25(OH)2D3500 ng/mL MeOH:H2O) of Data180801_VTD PFP_IPD 24,25(OH)2D3.wiff(

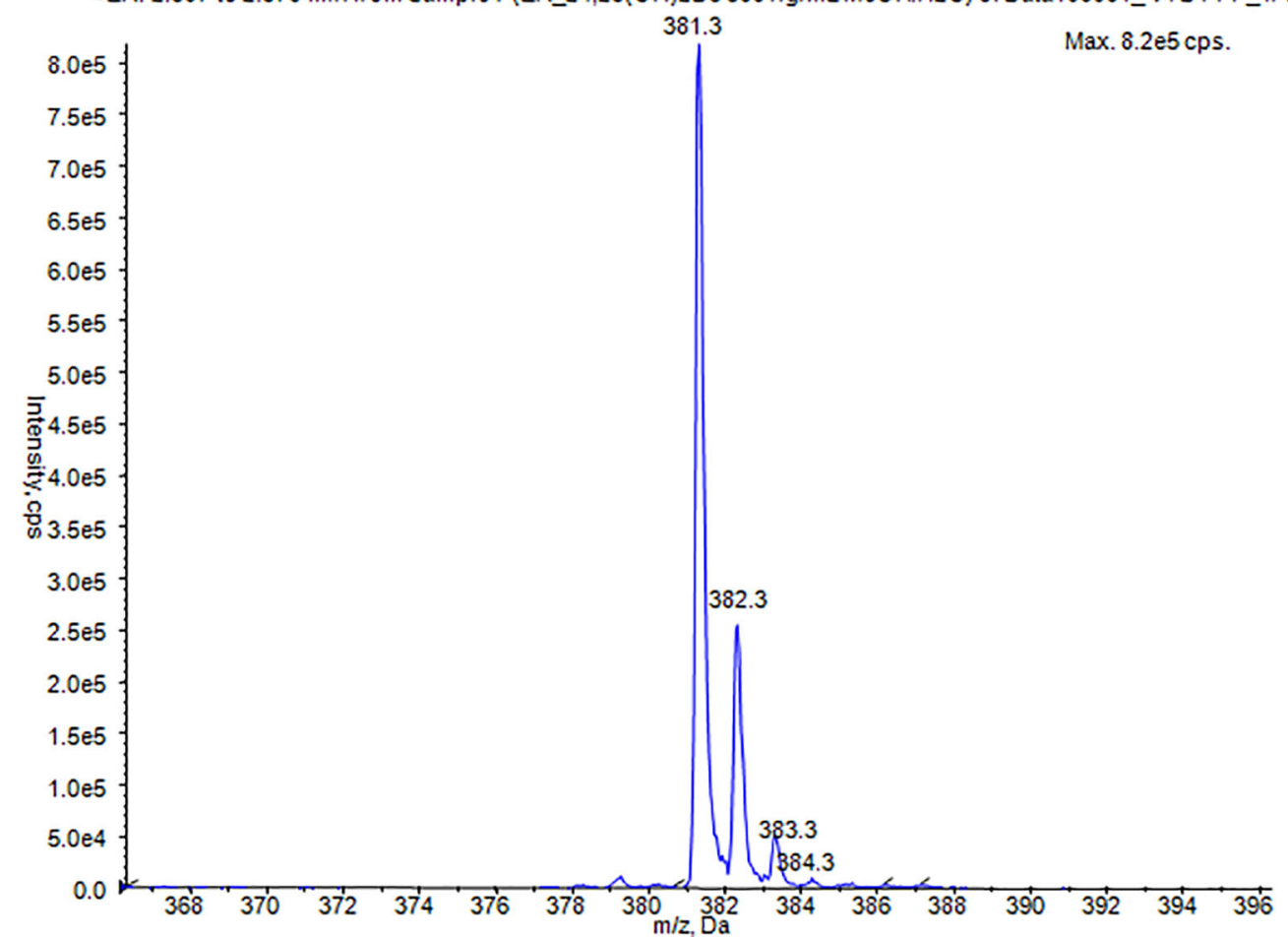

Figure 2 
Supplemental Table 1. Experimental isotopic abundances of 25(OH)D2

\begin{tabular}{cccc}
\hline & $\mathrm{m} / \mathrm{z}$ & & \multicolumn{2}{c}{ Experimental Abundances ${ }^{1}$} \\
Precursor & Product & $25(\mathrm{OH}) \mathrm{D} 2$ & ${ }^{2} \mathrm{H}_{3}-25(\mathrm{OH}) \mathrm{D} 2$ \\
\hline 395 & 377 & 0.7340 & - \\
\hline 396 & 378 & 0.2265 & - \\
\hline 397 & 377 & 0.0014 & 0.0326 \\
\hline 398 & 379 & 0.0339 & - \\
& 378 & 0.0004 & 0.7080 \\
\hline 399 & 380 & 0.0033 & 0.2201 \\
\hline & 381 & - & 0.0016 \\
& 380 & - & 0.0342 \\
& 382 & - & 0.0035 \\
\hline & 383 & - & \\
\hline
\end{tabular}

${ }^{1} 5$ injections of $0.5 \mu \mathrm{g} / \mathrm{mL}$ individual standards. 
Supplemental Table 2. Experimental isotopic abundances of 25(OH)D3

\begin{tabular}{cccc}
\hline & $\mathrm{m} / \mathrm{z}$ & & \multicolumn{2}{c}{ Experimental Abundances ${ }^{1}$} \\
Precursor & Product & $25(\mathrm{OH}) \mathrm{D} 3$ & ${ }^{2} \mathrm{H}_{6}-25(\mathrm{OH}) \mathrm{D} 3$ \\
\hline 383 & 365 & 0.7437 & - \\
\hline 384 & 366 & 0.2194 & - \\
\hline 385 & 365 & 0.0014 & - \\
\hline 386 & 367 & 0.0316 & - \\
\hline 387 & 366 & 0.0004 & 0.0093 \\
\hline 388 & 368 & 0.0028 & 0.0538 \\
\hline 389 & 369 & - & 0.7041 \\
\hline 390 & 370 & - & 0.2006 \\
\hline 391 & 371 & - & 0.0282 \\
& 372 & - & 0.0026 \\
\hline 373 & 374 & - & \\
\hline
\end{tabular}

${ }^{1} 5$ injections of $0.5 \mu \mathrm{g} / \mathrm{mL}$ individual standards. 
Supplemental Table 3. Experimental isotopic abundances of 24,25(OH)2D3

\begin{tabular}{cccc}
\hline & $\mathrm{m} / \mathrm{z}$ & & \multicolumn{2}{c}{ Experimental Abundances ${ }^{1}$} \\
Precursor & Product & $24,25(\mathrm{OH}) 2 \mathrm{D} 3$ & ${ }^{2} \mathrm{H}_{6}-24,25(\mathrm{OH}) 2 \mathrm{D} 3$ \\
\hline 381 & 363 & 0.738 & - \\
\hline 382 & 364 & 0.222 & \\
\hline 383 & 363 & 0.001 & - \\
& 365 & 0.034 & - \\
\hline 384 & 364 & 0.0004 & 0.036 \\
\hline 386 & 366 & 0.004 & 0.700 \\
\hline 387 & 368 & - & 0.003 \\
\hline 388 & 369 & - & 0.214 \\
\hline 389 & 369 & - & 0.033 \\
\hline
\end{tabular}

${ }^{1} 5$ injections of $0.5 \mu \mathrm{g} / \mathrm{mL}$ individual standards. 


\section{Re-certification of hydroxyvitamin D standards by isotope pattern deconvolution. Suplemental information.}

\section{IPD Detailed}

Notes on isotope pattern deconvolution (IPD) calculation. Part of this notes are provided by JI GarciaAlonso's “Enriched Stable Isotopes" research group. University of Oviedo. Spain.

IPD is an alternative calculation to classical calibration curve based procedure in Isotope Dilution Mass Spectrometry (IDMS). The addition of isotopically enriched analogues of the analytes under study produces an alteration of the natural distribution of isotopic abundances.

In IPD, the sample containing the natural abundance analyte is spiked with an exactly known amount of the isotope-labelled internal standard, which is traditionally enriched with ${ }^{13} \mathrm{C}$ or ${ }^{2} \mathrm{H}$. Thus, in the mixture we have molecules from the same compound showing two different isotope patterns: the natural abundance and the isotopically labeled. The isotope distribution of the molecule, or any fragment, in the mixture can be measured by low resolution Mass Spectrometry. Traditionally, the peak area ratio between two given masses in the mixture is measured for IDMS to build the calibration curve. In the IPD approach, the whole or part of the isotope pattern is measured. The total amount (mols) of compound in a given mixture, $N_{\text {mix }}$, can be distributed between the two sources by:

$N_{\text {mix }}=N_{\text {nat }}+N_{\text {lab }}$

where $N_{m i x}$ is the total amount of compound found in the mixture; $N_{\text {nat }}$ is the amount coming from the compound with natural isotope abundances; and, $N_{l a b}$ is the known amount of the labelled compound in the mixture coming from the spike added. Similar amount balances can be also obtained for all isotope compositions of the compound considered, as shown by equation [2], illustrated for a nominal mass $i$ as an example:

$N_{m i x}^{i}=N_{n a t}^{i}+N_{l a b}^{i}$

To conduct the calculations it is assumed that all possible isotope compositions possessing the same nominal mass are added together in the mass spectrum without differences in ionisation efficiencies or isotopic effects and are free of interferences. Then, equation [2] can be expressed as a linear combination of the total amount of each compound (natural abundance or labeled) and their corresponding isotope compositions:

$N_{\text {mix }} \cdot A_{m i x}^{i}=N_{\text {nat }} \cdot A_{\text {nat }}^{i}+N_{l a b} \cdot A_{\text {lab }}^{i}$

where $A_{m i x}^{i}$ is the experimentally measured relative abundance of the compound at nominal mass $i$ in the mixture; $A_{\text {nat }}^{i}$ is the theoretical relative abundance of the natural abundance compound at the 
same nominal mass and $A_{l a b}^{i}$ is the theoretical relative abundance in the isotopically labelled compound. When we divide equation [3] by equation [1], the following expression is obtained:

$A_{m i x}^{i}=x_{n a t} \cdot A_{n a t}^{i}+x_{l a b} \cdot A_{l a b}^{i}$

where

$$
x_{n a t}=\frac{N_{n a t}}{N_{n a t}+N_{l a b}}
$$

and

$$
x_{l a b}=\frac{N_{l a b}}{N_{n a t}+N_{l a b}}
$$

In expressions [5] and [6] the variables $x_{n a t}$ and $x_{l a b}$ indicate the molar fractions of the compound in the spiked sample arising from the two different sources: natural abundance or isotopically labelled. A typical organic compound may show different isotope compositions at $\boldsymbol{n}$ nominal masses so, equations for all these nominal masses can be obtained in a similar way to equation [4] and they can be expressed in matrix notation as:

$$
\left[\begin{array}{c}
A_{m i x}^{1} \\
A_{m i x}^{2} \\
A_{m i x}^{3} \\
\ldots \\
A_{m i x}^{n-1} \\
A_{m i x}^{n}
\end{array}\right]=\left[\begin{array}{cc}
A_{\text {nat }}^{1} & A_{l a b}^{1} \\
A_{\text {nat }}^{2} & A_{\text {lab }}^{2} \\
A_{\text {nat }}^{3} & A_{\text {lab }}^{3} \\
\ldots & \ldots \\
A_{n a t}^{n-1} & A_{l a b}^{n-1} \\
A_{n a t}^{n} & A_{l a b}^{n}
\end{array}\right] \cdot\left[\begin{array}{c}
x_{n a t} \\
x_{l a b}
\end{array}\right]+\left[\begin{array}{c}
e^{1} \\
e^{2} \\
e^{3} \\
\ldots \\
e^{n-1} \\
e^{n}
\end{array}\right]
$$

Where $A_{\text {mix }}^{i}$ are the experimentally measured relative abundance of the compound in the mixture for the different masses i; and $A_{\text {nat }}^{i}$ and $A_{\text {lab }}^{i}$ are the theoretical calculated (or experimentally measured) relative abundances of the natural and isotopically labeled compounds.

As we have more parameters (nominal masses) than unknowns (molar fractions) an error vector is included in equation [7]. The best values of $x_{n a t}$ and $x_{l a b}$ are found by least square minimization of the error vector "e".

Once the molar fractions of the compound are computed by the linear regression the amount of natural abundance compound can be calculated, as $N_{\text {lab }}$ is known, using the equation:

$$
\frac{N_{n a t}}{N_{l a b}}=\frac{x_{n a t}}{x_{l a b}}=R
$$

Equation [8] comes from dividing equation [5] by equation [6]. Note that equation [8] takes the form of straight line of slope 1 and intercept 0 when the ratio of mols $\left(N_{\text {nat }} / N_{\text {lab }}\right)$ is plotted against the ratio of molar fractions $\left(\mathrm{R}=X_{\text {nat }} / X_{l a b}\right)$, regardless of the labeling of the molecule. 
When tandem mass spectrometry (QqQ) in Selected Reaction Monitoring (SRM) mode is used, the different isotope compositions coming from the same nominal mass could generate fragment ions with various nominal masses, leading to a mass isotopomer distribution different from the original deprotonated ion. (Different isotopic composition with the same nominal mass are due to the presence of a ${ }^{13} \mathrm{C}$ atom or alternatively a ${ }^{15} \mathrm{~N}$ atom in the molecule, for example.)

If we assume that the fragmentation efficiency does not depend on the isotope composition, the relative abundance of any precursor ion will be distributed among their corresponding product ions. In other words, the sum of the relative abundances for all the transitions coming from the same nominal mass will be equivalent to the theoretical abundance of precursor ion.

Thus, the resulting isotopic composition $\left(A_{\text {mix }}^{S R M_{i}}\right)$, (Figure 1C in the manuscript) measured after mixing the natural occurring analytes in the samples and the spiked labelled analogues, is a linear combination of the natural $\left(A_{\text {nat }}^{S R M_{i}}\right)$ (Figure 1A) and enriched $\left(A_{\text {lab }}^{S R M_{i}}\right)$ (Figure 1B) compound's isotopic distribution of abundances.

For a single spike and $n$ measured transitions in the MS/MS system, this can be expressed as matrix notation as follows:

$$
\left[\begin{array}{c}
A_{\text {mix }}^{S R M_{1}} \\
A_{\text {mix }}^{S R M_{2}} \\
\vdots \\
A_{\text {mix }}^{S R M_{n}}
\end{array}\right]=\left[\begin{array}{cc}
A_{\text {nat }}^{S R M_{1}} & A_{\text {lab }}^{S R M_{1}} \\
A_{\text {nat }}^{S R M_{2}} & A_{\text {lab }}^{S R M_{2}} \\
\vdots & \vdots \\
S R M_{n} & S \\
A_{\text {nat }}^{S R M_{n}} & A_{\text {lab }}^{S S M_{n}}
\end{array}\right]\left[\begin{array}{l}
X_{\text {nat }} \\
X_{\text {lab }}
\end{array}\right]+\left[\begin{array}{c}
e^{S R M_{1}} \\
e^{S R M_{2}} \\
\vdots \\
e^{S R M_{n}}
\end{array}\right]
$$

As said before for MS mode, in order to solve the equation system, an error vector is included $\left(e^{S R M_{i}}\right)$. Minimization of this parameter by multiple linear regression gives the values of molar fractions $\left(X_{\text {nat }}\right.$ and $X_{\text {lab }}$ respectively) that best fit the experimental data of the mix. These calculations can be easily performed with the Excel "Linest" function (see an example in the Excel attached document), which uses the least squares method to calculate the line of best fit through a supplied set of " $y$ " (in this cases, $\left.A_{\text {mix }}^{S R M_{i}}\right)$ and "x" $\left(\left(A_{\text {nat }}^{S R M_{i}}\right)\right.$ and $\left.\left(A_{\text {lab }}^{S R M_{i}}\right)\right)$ values. Finally, since the amount of labelled compound $\left(N_{\text {lab }}\right)$ spiked into the sample is known, the amount of natural compound $\left(N_{\text {nat }}\right)$ is readily obtained:

$$
N_{\text {nat }}=N_{\text {lab }} \frac{X_{\text {nat }}}{X_{\text {lab }}}
$$

Note that equation [9] will provide an exact solution when $\boldsymbol{n}=2$ and a least squares solution when $\boldsymbol{n}>$ 2. Hence, the selection of the number of masses to be used in the calculations will depend on practical considerations. Three or four masses (or transitions in SRM mode) are typically measured when the method is finally applied with quantification purposes.

Previously to its aplication, this methodology requires the full characterization of both compounds (natural and labelled) in terms of their isotopomer distribution of abundances, i.e. calculating or measuring all the transitions to build matrix " $x$ ". In the present work isotopomer abundances have been experimentally measured. As said before, no methodological calibration is needed, and quantification is directly conducted in the sample after one injection. 
Example of IPD quantification for 25(OH)D3

First, the peak areas obtained for each transition are converted to abundances ( $\mathrm{Ab}$ mix) Then, the multiple linear regression is performed to obtain the molar fractions of natur As this calculation provides two values that do not comply with $\chi_{\mathrm{lab}}+\chi_{\mathrm{nat}}=1$, those value:

\begin{tabular}{ccccc}
\multicolumn{2}{c}{ Injection 1 } & \multicolumn{3}{c}{ Abundances } \\
$\mathrm{m} / \mathrm{z}$ & Area & Labelled & Natural & Ab mix \\
$383>365$ & $\mathbf{3 9 6 6 0 0 0}$ & 0 & 0,7437 & $\mathbf{0 , 3 7 8 3 2 7}$ \\
$384>366$ & $\mathbf{1 1 8 5 0 0 0}$ & 0 & 0,2194 & $\mathbf{0 , 1 1 3 0 4 0}$ \\
$389>371$ & $\mathbf{4 1 5 0 0 0 0}$ & 0,7041 & 0 & $\mathbf{0 , 3 9 5 8 7 9}$ \\
$390>372$ & $\mathbf{1 1 8 2 0 0 0}$ & 0,2006 & 0 & $\mathbf{0 , 1 1 2 7 5 4}$ \\
Sum & 10483000 & & &
\end{tabular}

Mw 25(OH)D3

400,6

Mw d6-25(OH)D3

406,6

Conc. Lab solution

$313,3 \mathrm{ng} / \mathrm{mL}$

Mass of solutions added to the vial Nat solution $0,0398 \mathrm{~g}$

Nat+Lab solutions

$0,0775 \mathrm{~g}$

Moles of labelled compound added to vial n d6-25(OH)D3 2,9049E-08 mol

Calculation of moles added to vial from the information provided by IPD $\chi$ Lab

0,52473112

$\chi$ Nat

0,47526888

N 25(OH)D3

2,6311E-08 mol

Conc. Nat solution $264,83 \mathrm{ng} / \mathrm{mL}$

This calculations are performed for each replicate and injection and the final concentra' 
1

al and labelled compounds.

s are normalized (black box).

\begin{tabular}{cc}
\multicolumn{2}{c}{ Linear Regression } \\
Natural & Labelled \\
0,509230535 & 0,562235958 \\
0,001250614 & 0,001324527 \\
0,999994219 & 0,00096971
\end{tabular}

Molar fractions $(\mathrm{x})$

Natural Labelled

$0,47526501 \quad 0,52473499$

tion value is calculated as the average \pm standard deviation. 
Information provided by linear regression

\begin{tabular}{cc}
\multicolumn{2}{c}{ Linear regression } \\
Nat & Lab \\
Xnat & Xlab \\
error Xnat & error Xlab \\
R2 & error Y
\end{tabular}

To perform calculations select cells $\mathrm{H} 11: 113$, where the results will be shown. (for example) Copy linear regression formula $=$ LINEST(F10:F13;D10:E13;0;1). (for example)

F10:F13 are known " $y$ " set of data. Experimental abundances in the mix D10:E13 are known "x" set of data. Abundances in the reference matrix ";0" regression passes through the origin ";1" gives statistics

Press simultaneously "Ctrl" + "Shift" + "Enter" to obtain results

Please, look at the inverted column order to introducing and showing data and calculation results 